\title{
Immune Checkpoint Inhibitors: Therapeutic Tools for Breast Cancer
}

\author{
Min Su, Chun-Xia Huang, Ai-Ping Dai*
}

\begin{abstract}
Breast cancer is one of the major threats to female health, and its incidence is rapidly increasing in many countries. Currently, breast cancer is treated with surgery, followed by chemotherapy or radiation therapy, or both. However, a substantial proportion of breast cancer patients might have a risk for local relapse that leads to recurrence of their disease and/or metastatic breast cancer. Therefore searching for new and potential strategies for breast cancer treatment remains necessary. Immunotherapy is an attractive and promising approach that can exploit the ability of the immune system to identify and destroy tumors and thus prevent recurrence and metastatic lesions. The most promising and attractive approach of immunotherapeutic research in cancer is the blockade of immune checkpoints. In this review, we discuss the potential of certain inhibitors of immune checkpoints, such as antibodies targeting cytotoxic T-lymphocyte antigen 4 (CTLA-4), programmed death 1 (PD-1) and lymphocyte activation gene-3 (LAG-3), in breast cancer therapeutics. Immune checkpoint inhibitors may represent future standards of care for breast cancer as monotherapy or combined with standard therapies.
\end{abstract}

Keywords: Breast cancer - immunotherapy - antibodies - cytotoxic t-lymphocyte antigen 4 - PD-1 - LAG-3

Asian Pac J Cancer Prev, 17 (3), 905-910

\section{Introduction}

Breast cancer is the most common invasive cancer and one of the leading causes of death among women in the world over (Ramsay et al., 2005; Rezaeian et al., 2014). It develops from breast tissue, specifically cells from the lining of lactiferous ducts and the lobules that supply the ducts with milk in a lactating female. The first noticeable symptom of breast cancer is typically a lump in the breast that feels different from the rest of the breast tissue. Other commonly mentioned symptoms may include: pain/soreness in part of the breast, a change in breast shape, the nipple may become inverted, discharge from nipple and skin puckering or dimpling (Jones et al., 2010). Breast cancer is a multi-factorial disease process affected by multiple risk factors such as age, sex, genetics, higher levels of certain hormones, certain dietary patterns and obesity (Blackburn et al., Rock et al., 2004). The incidence of breast cancer is rapidly increasing, especially in developing countries due to the lack of awareness in women for its early detection and the lack of a formal screening program, inadequate and inappropriate treatment because of limited financial resources (Agarwal et al., 2009). American Cancer Society provided an overview of female breast cancer statistics which estimated approximately 232,340 new cases of invasive breast cancer and 39,620 breast cancer deaths among US women in 2013 (DeSantis et al., 2014).

The most commonly used screening methods of breast cancer are: clinical breast examination (CBE), breast self examination (BSE) and mammography (Saslow et al., 2004; Gürsoy et al., 2009). Breast cancer is most commonly treated with surgery, which may be followed by chemotherapy or radiation therapy, or both. Although the conventional treatments for breast cancer are sometimes beneficial, a substantial proportion of breast cancer patients might have a risk for local relapse that leads to develop a recurrence of their disease and/or metastatic breast cancer (Ghochikyan et al., 2014). For this reason, investigating new and potential strategies for breast cancer treatment remains necessary. Recently, immunotherapy is represented as an attractive and promising approach that can exploit the ability of the immune system to identify and destroy tumors to prevent the recurrence and metastatic lesions of breast cancer (Ascierto and Marincola, 2014).

\section{Immunotherapies in cancer}

The immune system plays a vital protective role against cancer by identifying and eliminating tumor cells (Ascierto and Marincola, 2014). Cancer cells express a variety of tumor associated antigens (TAA) which can be recognized by a special type of immune cell known 
as cytotoxic $\mathrm{T}$ cell to induce an immune response against cancer (Murala et al., 2010). However, the immune system can not always recognize the specific target or the response might not be strong enough to destroy the cancer. Sometimes cancer cells take advantage of the ability to hide from the immune system by exploiting a series of immune escape mechanisms that might be responsible for the failure of the immune system to destroy or control cancer (Wright, 2012; Criscitiello and Curigliano, 2013). Immunotherapy may be viewed as a promising approach to anticancer treatment which has been reported to overcome tumor-induced immune dysfunction and restore the ability of the immune system to fight cancer (Shore, 2015; Rothschild et al., 2015).

Recent immune based therapeutic approaches for cancer are: therapeutic cancer vaccines, cytokines, immune adjutants, immune checkpoint inhibitors and adoptive T cell therapy (ACT) (Wang et al., 2015; Ernst and Anderson, 2015). Therapeutic cancer vaccines contain a specific antigen expressed on tumor cell which may induce specific antitumor immune response of the patients through the introduction of tumor antigens (Rothschild et al., 2015; Hao et al., 2012; Schlom, 2012). Adoptive T cell transfer is designed to infuse tumor-specific cytotoxic $\mathrm{T}$ cells into cancer patients which can recognize, target and destroy tumor cells (Perica et al., 2015). The other promising immunotherapeutic approach such as immune checkpoint inhibitors target specific immune regulatory checkpoints to overcome tumor-induced immune dysfunction and to enhance immune response against cancer (Ascierto and Marincola, 2014; Rothschild et al., 2015).

The most promising and attractive approach of immunotherapeutic research in cancer is the blockade of immune checkpoints (Pardoll, 2012). Immune checkpoints are the $\mathrm{T}$ lymphocytes activation modulators that are crucial for maintaining self-tolerance and modulating the duration and amplitude of physiological immune responses in peripheral tissues in order to prevent uncontrolled immune reactions and host tissue damage. But cancer cells are able to escape from the immune system mainly by hijacking the immune cell-intrinsic checkpoints that induce the tumor cells to evade the host immune system by inhibiting T lymphocytes activation (Criscitiello and Curigliano, 2013; Ileana et al., 2013). Blocking of these immune checkpoints such as cytotoxic T-lymphocyte antigen 4 (CTLA-4), programmed death 1 (PD-1), PD ligand 1 (PD-L1) and lymphocyte activation gene-3 (LAG-3) with monoclonal antibodies (mAbs) or agents that are immunostimulatory can modulate the immunosuppressive environment, augment anti-tumor immunity and induce tumor regression (Stagg and Allard, 2013; Ghochikyan et al., 2014).

\section{Immune checkpoint inhibitors in breast cancer treatment}

The use of immune checkpoint inhibitors is a promising cancer therapeutic strategy which aims at blocking specific immune regulatory checkpoints to enhance the endogenous antitumor immunity to produce

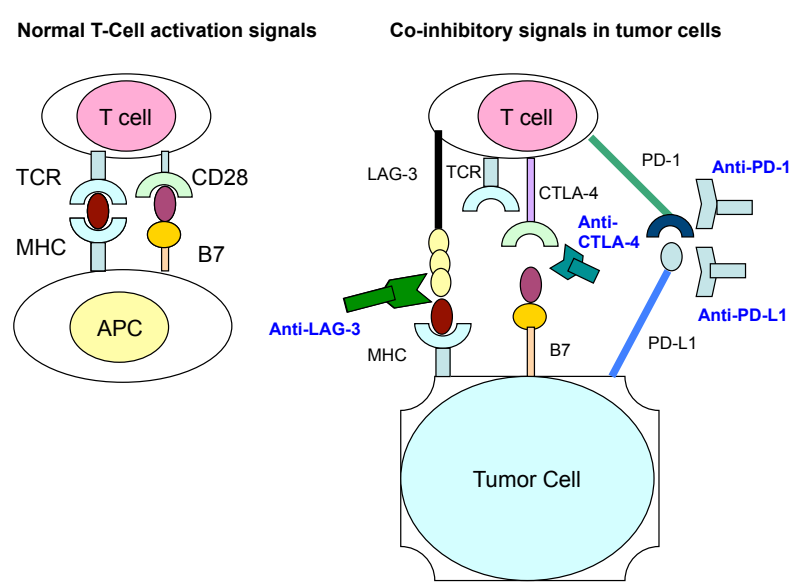

Figure 1. Blockade of Immune Checkpoints with Antibodies in Cancer Immunotherapy. During T cell activation, $\mathrm{T}$ cells recognize major histocompatibility complex (MHC) complex through T cell receptor (TCR) on antigenpresenting cells (APCs) via an activation signal provided by the interaction between CD28 and B7 (Sharma et al., 2011; Rudd et al., 2009). Shortly after T cell activation, cytotoxic T lymphocyte-associated protein-4 (CTLA-4) competes with CD28 for binding to B7 and transmits an inhibitory signal to turn off the immune response by suppressing T cell activation (Chen and Flies, 2013; Carreno et al., 2000). Another receptor molecule that delivers inhibitor signals is programmed death-1 (PD-1) which is expressed on activated T cells after antigen exposure. PD-1 provides inhibitory signals to activated $\mathrm{T}$ cell via interaction with PD-L1 which is expressed on tumor cells (Pedoeem et al., 2014; Gatalica et al., 2014). Lymphocyte-activation gene 3 (LAG-3) is another recently identified inhibitory receptor that is up-regulated on some epithelial cancers. LAG-3 provides inhibitory signal to activated T cells via LAG-3/MHC class II interaction which down-regulates T-cell mediated responses (Triebel, 2003; Gandhi et al., 2006). Blockade of the immune inhibitor molecules with antibodies can therapeutically block the inhibitory signal which results in the preferential activation of $T$ cells and restore immune activity in the tumor micro-environment (Goldberg and Darke, 2011; Brahmer et al., 2012; Blank, 2014)

durable responses in multiple cancers including breast cancer (Stagg and Allard, 2013; Shin and Ribas, 2015; Sharma et al., 2011). This review article summarizes the mechanism of actions and the therapeutic use of checkpoint inhibitors in the treatment of breast cancer with subsequent clinical studies. Figure 1 presents a generalized mechanism.

\section{Antibodies targeting cytotoxic T-lymphocyte antigen 4 (CTLA-4)}

Cytotoxic T-lymphocyte antigen 4 (CTLA-4, also known as CD152) is the most well known and the first clinically validated immune checkpoint which is expressed on the surface of the effector $\mathrm{T}$ cells (Teff cells) (Wang et al., 2015; Yano et al., 2014). It belongs to the immunoglobulin (Ig) superfamily and regulates the amplitude of the early stages of $T$ cell activation by down-modulating the activity of Teff cells and enhancing the cell immunosuppressive activity of CD25+FOXP3+T regulatory cells (Tregs) (Jago et al., 2004; Takahashi et al., 2000). T cell activation is initiated by stimulation of T cell receptor (TCR) through the recognition of peptide major 
histocompatibility complexes (MHC) (Sharma et al., 2011). CTLA-4 is thought to counteract the co-stimulatory signal from its homologue, CD28 by competitively binding on the T cell surface to B7 proteins, B7-1 (CD80) and B7-2 (CD86) on the antigen-presenting cell (APC) (Qureshi et al., 2011; Rudd et al., 2009). CTLA-4 transmits an inhibitory signal to turn off the immune response by suppressing T cell activation and promoting the function of regulatory T cells (Tregs) which can dampen the immune response at the tumor site (Chen and Flies, 2013; Carreno et al., 2000). Blockade of CTLA4 with anti-CTLA4 antibodies enhances the anti-tumor function of Teff cells that can migrate to and attack the tumor. CTLA-4 blocking antibodies also induce Treg depletion by inhibiting CTLA4-mediated signaling pathway through $\mathrm{T}$ cell proliferation and tumor-specific cytotoxicity (Blank, 2014; Selby et al., 2013). Anti-CTLA4 therapy represents a promising and novel approach for the treatment of various types of cancers, including breast cancer. Two CTLA-4 targeting antibodies such as ipilimumab and tremelimumab have been developed to promote T-cell activation and antitumor immunity in clinical trials (Poust, 2008).

Ipilimumab (also known as MDX-010 and marketed as Yervoy) is a fully humanized monoclonal antibody (IgG1) against CTLA-4 that has shown activity in patients with previously treated metastatic melanoma (Hodi et al., 2010). It is also being evaluated in breast cancer patients. A clinical trial of preoperative cryoablation (a process that uses extreme cold temperatures through the needle to kill the cancer cells) or ipilimumab alone, or given together in patients with early stage breast cancer is in progress [ClinicalTrials.gov, NCT01502592].

Tremelimumab (formerly known as ticilimumab) is a fully human IgG2 monoclonal antibody against CTLA-4 which stimulates the immune systems to attack the tumor cells. It has induced durable tumor responses with manageable toxicities in patients with advanced melanoma in a Phase I/II clinical trial (Reuben et al., 2006). However, as a single agent, tremelimumab has not been evaluated in patients with breast cancer. For the patients with breast cancer, hormonal therapy may be well suited for combination with anti-CTLA4 mAb. The combination of tremelimumab and exemestane (steroidal aromatase inhibitor that inhibits the synthesis of estrogen) was tested to evaluate the safety and preliminary efficacy for patients with hormone-responsive metastatic breast cancer in a phase I trial which tested the maximum tolerated dose of tremelimumab with exemestane was $6 \mathrm{mg} / \mathrm{kg}$ every 90 days (Vonderheide et al., 2010). The combination of local radiation therapy (RT) with CTLA-4 blockade is a promising immunotherapeutic strategy which can induce therapeutically significant antitumor immunity. In an animal experiment, the poorly immunogenic metastatic mouse mammary carcinoma 4T1 was used as a model of breast cancer and mice were treated with RT $+9 \mathrm{H} 10$ (monoclonal antibody against CTLA-4). The combination of RT to the primary tumor with CTLA-4 elicited CD8+ T cell-dependent antitumor immunity which can inhibit the growth of spontaneous lung metastases (Demaria et al., 2005; Pilones et al., 2009). Hurwitz et al. (1998) demonstrated that certain tumors, such as the SM1 mammary carcinoma remain refractory to the administration of anti-CTLA-4 antibody as a single treatment. They treated SM1 tumor with a granulocyte-macrophage colony-stimulating factor (GMCSF)-expressing vaccine followed by anti-CTLA-4 which resulted in long-lasting immunity to SM1 and regression of SM1 cell line-induced mammary carcinoma.

\section{Antibodies targeting programmed death 1 (PD-1)}

Programmed death-1 (PD-1, also known as CD279) is another transmembrane molecule belongs to CD28 Ig superfamily (Intlekofer and Thompson, 2013). PD-1 is expressed on activated and exhausted $\mathrm{T}$ cells during thymic development. It provides co-inhibitory signals that reduces cytokine production and control $\mathrm{T}$ cell responses in peripheral tissues via interaction with its ligands, PD-L1 (B7-H1, CD274) and PD-L2 (B7-DC, CD273) (Keir et al., 2008; Pedoeem et al., 2014). When PD-1 binds with cancer cells bearing one of its ligands (PD-L1/PD-L2), immune-modulatory $\mathrm{T}$ cell activity is attenuated which facilitates disease progression (Gatalica et al., 2014). Blocking interactions between PD-1:PD-L1/ PD-L2 with monoclonal antibodies could be an effective anti-cancer therapy which can restore immune activity in the tumor micro-environment (Rothschild et al., 2015; Brahmer et al., 2012). It is reported that blockade of PD-1/PD-L1 signaling could also promote dendritic cell (DC) maturation, augment DC primed T cell response and prevent tumor cell dampened $\mathrm{T}$ cell inactivation $(\mathrm{Ge}$ et al., 2013). Several PD-1/PD-L1 targeting monoclonal antibodies have been developed to regulate tumor-specific T-cell expansion in patients with malignant melanoma and various other solid tumor types (Hao et al., 2014).

Nivolumab (formerly known as BMS-936558 or MDX-1106) is a fully human $\mathrm{IgG} 4$ monoclonal PD-1 targeting antibody that inhibits the interaction of PD-1 with its ligands and thereby, enhances antitumor immunity (Weber et al., 2013). A clinical trial of nivolumab with nab-paclitaxel (albumin-bound paclitaxel, trade name Abraxane) in patients with recurrent metastatic breast cancer is currently underway [ClinicalTrials.gov, NCT02309177].

Pembrolizumab (formerly known as MK-3475 and lambrolizumab) is a humanized monoclonal IgG4-kappa isotype designed to directly block the interaction between PD-1 and its ligands, PD-L1 and PD-L2. Pembrolizumab showed significant anti-cancer activity in patients with metastatic melanoma and different types of cancer (Hamid et al., 2013). A Phase I/II trial evaluating the efficacy of pembrolizumab and trastuzumab (a monoclonal antibody that interferes with the HER2/neu receptor) in patients with trastuzumab-resistant, HER2-positive metastatic breast cancers is under investigation [ClinicalTrials.gov, NCT02129556].

Karyampudi et al. (2014) reported that the combination of anti-PD-1 antibody and a multipeptide vaccine (consisting of immunogenic peptides derived from breast cancer antigens, neu, legumain, and $\beta$-catenin) would enhance the anti-cancer immunity in a murine model of 
breast cancer. They observed that the combination therapy regimen enhanced vaccine efficacy for the treatment of breast cancer-bearing mice by altering both CD8 T cell and DC components of the tumor microenvironment. Ge et al. (2013) reported that blockade of PD-1/PD-L1 immune checkpoint during DC vaccination induces potent protective immunity by preventing tumor growth and prolonging survival times in a breast tumor-bearing huSCID mice model. In a phase 1 study, anti-PD-L1 antibody (BMS-936559, Bristol-Myers Squibb) was administered to a total 207 patients in whom 4 patients were with breast cancer. The safety and efficacy of BMS-936559 was evaluated and none of the four breast cancer patients treated had a response might be due to the small number of patients or they might have had PD-L1 negative breast cancer (Brahmer et al., 2012; Janakiram et al., 2012).

\section{Antibodies targeting lymphocyte activation gene-3 (LAG-3)}

Lymphocyte-activation gene 3 (LAG-3, also known as CD223) is another recently identified inhibitory receptor that is highly expressed on TReg cells and anergic $\mathrm{T}$ cells (Triebel, 2003). In 2005, it was defined as immune checkpoint as it provides inhibitory signal to activated effector $\mathrm{T}$ cells and augment the suppressive activity of TReg cells (Pardoll, 2012). MHC class II is the only known ligand for LAG3 which is up-regulated on some epithelial cancers and LAG-3/MHC class II interaction down-regulates T-cell mediated responses (Triebel, 2003; Gandhi et al., 2006). Blockade of LAG-3/MHC class II interaction with monoclonal antibodies enhances tumorspecific CD8+ T cell expansion and cytokine production which is being evaluated in a number of clinical trials in cancer patients (Macon-Lemaître and Triebel, 2005; Goldberg and Drake, 2011). However, blockade of LAG-3 with monoclonal antibodies is not studied well in patients with breast cancer. A phase I/II study was conducted in which patients with metastatic breast carcinoma (MBC) were received first-line paclitaxel and IMP321 (a recombinant soluble LAG-3Ig fusion protein). In this study, a fixed dose of paclitaxel $\left(80 \mathrm{mg} / \mathrm{m}^{2}\right.$ at D1, D8 and D15 of a 4-week cycle) and three IMP321 doses (0.25, 1.25 and $6.25 \mathrm{mg}$ at D2 and D16 of this 4-week cycle, for 6 courses) were administered. The combination of paclitaxel and IMP321 (LAG-3Ig) enhances immune responses and antitumor activity by inducing APC activation (monocytes and dendritic cells) and increasing the percentage of cytotoxic effector-memory CD8 T cells (Brignone et al., 2010). An animal study was conducted in which soluble mouse LAG-3 fusion protein (mLAG-3Ig) was administered in combination with DNA vaccination in HER-2/neu Transgenic BALB/c Mice. This combined vaccination elicited a stronger and effective protection, reduced carcinoma multiplicity and a marked expansion of CD8+/CD11b+/CD28+ effector and CD8+/CD11b+/ CD28- memory effector T-cell populations in immunized mice (Cappello et al., 2003).

\section{Limitations of the use of checkpoint inhibitors}

Immune checkpoint blocking antibodies mediates durable tumor remission and effectively induces long standing anti-tumor immunity (Janakiram et al., 2012). However, the development of checkpoint inhibitor immunotherapy as a treatment modality for cancer has been hampered by several factors such as eligibility of the participants in clinical trial, the methods of evaluation, financial support, appropriate selection of the optimal dose and schedule, and toxicities of the immunotherapeutic agents (Pardoll, 2012; Lesterhuis et al., 2011). The use of checkpoint inhibitors may induce frequent drugrelated adverse events including diarrhea, rash, fatigue, nausea, pruritus, headache, pneumonitis, hypothyroidism, vitiligo, colitis, hepatitis, hypophysitis, sarcoidosis, endophthalmitis, diabetes mellitus, liver dysfunction and myasthenia gravis (Dolan and Gupta, 2014; Kong et al., 2010). The frequency of adverse events of different checkpoint inhibitors is different which might be due to the distinct biologic features (Wang et al., 2015).

\section{Conclusions}

The treatment of breast cancer remains challenging as the cure rates for metastatic breast cancer is dismal. Targeting the immune checkpoint molecules by antibodies is an attractive therapeutic strategy for breast cancer which can modulate the antitumor immune response with manageable toxicity. These therapies may represent the future standards of care for breast cancer as monotherapy or combined with standard therapies. Understanding the pathological mechanisms of different checkpoint molecules involved in cancer progression, immunerelated toxicities and the mechanisms of immunologic resistance to checkpoint modulation may increase the efficacy of cancer immunotherapies with its potential clinical applications.

\section{Acknowledgements}

This study was supported by grant form Foundation of the Changsha Medical University (2014) and the construct program of the key discipline in Hunan province.

\section{References}

Agarwal G, Ramakant P, Forgach ER, et al (2009). Breast cancer care in developing countries. World J Surg, 33, 2069-76.

Ascierto PA, Marincola FM (2014). What have we learned from cancer immunotherapy in the last 3 years? J Transl Med, 12, 141.

Blackburn GL, Wang KA (2007). Dietary fat reduction and breast cancer outcome:results from the Women's Intervention Nutrition Study (WINS). Am J Clin Nutr, 86, 878-81.

Blank CU (2014). The perspective of immunotherapy: new molecules and new mechanisms of action in immune modulation. Curr Opin Oncol, 26, 204-14.

Brahmer JR, Tykodi SS, Chow LQ, et al (2012). Safety and activity of anti-PD-L1 antibody in patients with advanced cancer. $N$ Engl J Med, 366, 2455-65.

Brignone C, Gutierrez M, Mefti F, et al (2010). First-line chemoimmunotherapy in metastatic breast carcinoma: 
combination of paclitaxel and IMP321 (LAG-3Ig) enhances immune responses and antitumor activity. J Transl Med, 8,71 .

Cappello P, Triebel F, Iezzi M, et al (2003). LAG-3 enables DNA vaccination to persistently prevent mammary carcinogenesis in HER-2/neu transgenic BALB/c mice. Cancer Res, 63 2518-25.

Carreno BM, BennettF, Chau TA, et al (2000). CTLA-4 (CD152) can inhibit $\mathrm{T}$ cell activation by two different mechanisms depending on its level of cell surface expression. J Immunol, 165, 1352-6.

Chen L, Flies DB (2013). Molecular mechanisms of T cell costimulation and co-inhibition. Nat Rev Immunol, 13, 227-42.

Criscitiello C, Curigliano G (2013). Immunotherapeutics for breast cancer. Curr Opin Oncol, 25, 602-8.

Demaria S, Kawashima N, Yang AM, et al (2005). Immunemediated inhibition of metastases after treatment with local radiation and CTLA-4 blockade in a mouse model of breast cancer. Clin Cancer Res, 11, 728-34.

DeSantis C, Ma J, Bryan L, Jemal A (2014). Breast cancer statistics, 2013. CA Cancer J Clin, 64, 52-62.

Dolan DE, Gupta S (2014). PD-1 pathway inhibitors: changing the landscape of cancer immunotherapy. Cancer Control, 21, 231-7.

Ernst B, Anderson KS (2015). Immunotherapy for the treatment of breast cancer. Curr Oncol Rep, 17, 426.

Gandhi MK, Lambley E, Duraiswamy J, et al (2006). Expression of LAG-3 by tumor-infiltrating lymphocytes is coincident with the suppression of latent membrane antigen-specific CD8+ T-cell function in Hodgkin lymphoma patients. Blood, 108, 2280-9.

Gatalica Z, Snyder C, Maney T, et al (2014). Programmed cell death 1 (PD-1) and its ligand (PD-L1) in common cancers and their correlation with molecular cancer type. Cancer Epidemiol Biomarkers Prev, 23, 2965-70.

Ge Y, Xi H, Ju S, Zhang X (2013). Blockade of PD-1/PD-L1 immune checkpoint during DC vaccination induces potent protective immunity against breast cancer in hu-SCID mice. Cancer Lett, 336, 253-9.

Ghochikyan A, Pichugin A, Bagaev A, et al (2014). Targeting TLR-4 with a novel pharmaceutical grade plant derived agonist, Immunomax ${ }^{\circledR}$, as a therapeutic strategy for metastatic breast cancer. $J$ Transl Med, 12, 322.

Goldberg MV, Drake CG (2011). LAG-3 in Cancer Immunotherapy. Curr Top Microbiol Immunol, 344, 269-78.

Gürsoy AA, Ylmaz F, Nural N, et al (2009). A different approach to breast self-examination education: daughters educating mothers creates positive results in Turkey. Cancer Nurs, 32, 127-34.

Hamid O, Robert C, Daud A, et al (2013). Safety and tumor responses with lambrolizumab (anti-PD-1) in melanoma. $N$ Engl J Med, 369, 134-44.

Hao MZ, Zhou WY, Du XL, et al (2014). Novel anti-melanoma treatment: focus on immunotherapy. Chin J Cancer, 33, 458-65.

Hodi FS, O'Day SJ, McDermott DF, et al (2010). Improved survival with ipilimumab in patients with metastatic melanoma. $N$ Engl J Med, 363, 711-23.

Hurwitz AA, Yu TF, Leach DR, Allison JP (1998). CTLA-4 blockade synergizes with tumor-derived granulocytemacrophage colony-stimulating factor for treatment of an experimental mammary carcinoma. Proc Natl Acad Sci U $S A, 95,10067-71$.

Ileana E, Champiat S, Soria JC (2013). Immune-checkpoints: the new anti-cancer immunotherapies. Bull Cancer, 100, 601-10.

Intlekofer AM, Thompson CB (2013). At the bench: preclinical rationale for CTLA-4 and PD-1 blockade as cancer immunotherapy. J Leukoc Biol, 94, 25-39.

Jago CB, Yates J, Câmara NO, Lechler RI, Lombardi G (2004). Differential expression of CTLA-4 among T cell subsets. Clin Exp Immunol, 136, 463-71.

Janakiram M, Abadi YM, Sparano JA, Zang X (2012). T cell coinhibition and immunotherapy in human breast cancer. Discov Med, 14, 229-36.

Jones SC, Gregory P, Nehill C, et al (2010). Australian women's awareness of breast cancer symptoms and responses to potential symptoms. Cancer Causes Control, 21, 945-58.

Karyampudi L, Lamichhane P, Scheid AD, et al (2014). Accumulation of memory precursor CD8 T cells in regressing tumors following combination therapy with vaccine and anti-PD-1 antibody. Cancer Res, 74, 2974-85.

Keir ME, Butte MJ, Freeman GJ, Sharpe AH (2008). PD-1 and its ligands in tolerance and immunity. Апnu Rev Immunol, 26, 677-704.

Kong YC, Wei WZ, Tomer Y (2010). Opportunistic autoimmune disorders: from immunotherapy to immune dysregulation. Ann N Y Acad Sci, 1183, 222-36.

Lesterhuis WJ, Haanen JB, Punt CJ (2011). Cancer immunotherapy--revisited. Nat Rev Drug Discov, 10, 591600.

Maçon-Lemaître L, Triebel F (2005). The negative regulatory function of the lymphocyte-activation gene-3 co-receptor (CD223) on human T cells. Immunol, 115, 170-8.

Murala S, Alli V, Kreisel D, Gelman AE, Krupnick AS (2010). Current status of immunotherapy for the treatment of lung cancer. J Thorac Dis, 2, 237-44.

Pardoll DM (2012). The blockade of immune checkpoints in cancer immunotherapy. Nat Rev Cancer, 12, 252-64.

Pedoeem A, Azoulay-Alfaguter I, Strazza M, Silverman GJ, Mor A (2014). Programmed death-1 pathway in cancer and autoimmunity. Clin Immunol, 153, 145-52.

Perica K, Varela JC, Oelke M, Schneck J (2015). Adoptive T cell immunotherapy for cancer. Rambam Maimonides Med $J, 6,4$.

Pilones KA, Kawashima N, Yang AM, et al (2009). Invariant natural killer $\mathrm{T}$ cells regulate breast cancer response to radiation and CTLA-4 blockade. Clin Cancer Res, 15, 597-606.

Poust J (2008). Targeting metastatic melanoma. Am J Health Syst Pharm, 65, 9-15.

Qureshi OS, Zheng Y, Nakamura K, et al (2011). Transendocytosis of CD80 and CD86: a molecular basis for the cell-extrinsic function of CTLA-4. Science, 332, 600-3.

Ramsay DT, Kent JC, Hartmann RA, Hartmann PE (2005). Anatomy of the lactating human breast redefined with ultrasound imaging. J Anat, 206, 525-34.

Reuben JM, Lee BN, Li C, et al (2006). Biologic and immunomodulatory events after CTLA-4 blockade with ticilimumab in patients with advanced malignant melanoma. Cancer, 106, 2437-44.

Rezaeian M, Sharifirad G, Mostafavi F, Moodi M, Abbasi MH (2014). The effects of breast cancer educational intervention on knowledge and health beliefs of women 40 years and older, Isfahan, Iran. J Educ Health Promot, 3, 43.

Rock CL, Flatt SW, Thomson CA, et al (2004). Effects of a high-fiber,low-fat diet intervention on serum concentrations of reproductive steroid hormones in women with a history of breast cancer. J Clin Oncol, 22, 2379-87.

Rothschild SI, Thommen DS, Moersig W, Müller P, Zippelius A (2015). Cancer immunology - development of novel anticancer therapies. Swiss Med Wkly, 145, 14066.

Rudd CE, Taylor A, Schneider H (2009). CD28 and CTLA-4 coreceptor expression and signal transduction. Immunol Rev, 229, 12-26. 


\section{Min Su et al}

Saslow D, Hannan J, Osuch J, et al (2004). Clinical breast examination: practical recommendations for optimizing performance and reporting. CA Cancer J Clin, 54, 327-44.

Schlom J (2012). Therapeutic cancer vaccines: current status and moving forward. J Natl Cancer Inst, 104, 599-613.

Selby MJ, Engelhardt JJ, Quigley M, et al (2013). Anti-CTLA-4 antibodies of $\mathrm{IgG} 2 \mathrm{a}$ isotype enhance antitumor activity through reduction of intratumoral regulatory T cells. Cancer Immunol Res, 1, 32-42.

Sharma P, Wagner K, Wolchok JD, Allison JP (2011). Novel cancer immunotherapy agents with survival benefit: recent successes and next steps. Nat Rev Cancer, 11, 805-12.

Shin DS, Ribas A (2015). The evolution of checkpoint blockade as a cancer therapy: what's here, what's next? Curr Opin Immunol, 33, 23-35.

Shore ND (2015). Advances in the understanding of cancer immunotherapy. BJU Int , 116, 321-9.

Stagg J, Allard B (2013). Immunotherapeutic approaches in triple-negative breast cancer: latest research and clinical prospects. Ther Adv Med Oncol, 5, 169-81.

Takahashi T, Tagami T, Yamazaki S, et al (2000). Immunologic self-tolerance maintained by CD25(+)CD4(+) regulatory $\mathrm{T}$ cells constitutively expressing cytotoxic $\mathrm{T}$ lymphocyteassociated antigen 4. J Exp Med, 192, 303-10.

Triebel F (2003). LAG-3: a regulator of T-cell and DC responses and its use in therapeutic vaccination. Trends Immunol, 24, 619-22.

Vonderheide RH, LoRusso PM, Khalil M, et al (2010). Tremelimumab in combination with exemestane in patients with advanced breast cancer and treatment-associated modulation of inducible costimulator expression on patient T cells. Clin Cancer Res, 16, 3485-94.

Wang DH, Guo L, Wu XH (2015). Checkpoint inhibitors in immunotherapy of ovarian cancer. Tumor Biol, 36, 33-9.

Weber JS, Kudchadkar RR, Yu B, et al (2013). Safety, efficacy, and biomarkers of nivolumab with vaccine in ipilimumabrefractory or -naive melanoma. J Clin Oncol, 31, 4311-8.

Wright SE (2012). Immunotherapy of breast cancer. Expert Opin Biol Ther, 12, 479-90.

Yano H, Thakur A, Tomaszewski EN, et al (2014). Ipilimumab augments antitumor activity of bispecific antibody-armed $\mathrm{T}$ cells. J Transl Med, 12, 191. 